\title{
Genomic Analysis and Lineage
} Identification of SARS-CoV-2 Strains in Migrants Accessing Europe Through the Libyan Route

\section{OPEN ACCESS}

Edited by:

Chaminda Jayampath Seneviratne, National Dental Centre of

Singapore, Singapore

Reviewed by: Massimo Ciccozzi,

Campus Bio-Medico University, Italy Mohamed Ali Daw,

University of Tripoli, Libya

*Correspondence: Walter Mazzucco walter.mazzucco@unipa.it

${ }^{\dagger}$ These authors have contributed equally to this work

Specialty section: This article was submitted to Infectious Diseases 96 Surveillance, Prevention and Treatment, a section of the journa Frontiers in Public Health

Received: 23 November 2020 Accepted: 15 February 2021 Published: 15 April 2021

Citation:

Tramuto F, Reale S, Lo Presti A, Vitale F, Pulvirenti C, Rezza G, Vitale F,

Purpari G, Maida CM, Zichichi S,

Scibetta S, Mazzucco W and Stefanelli P (2021) Genomic Analysis and Lineage Identification of

SARS-CoV-2 Strains in Migrants Accessing Europe Through the Libyan Route. Front. Public Health 9:632645.

doi: 10.3389/fpubh.2021.632645

\author{
Fabio Tramuto ${ }^{1,2 \dagger}$, Stefano Reale ${ }^{3 \dagger}$, Alessandra Lo Presti ${ }^{4 \dagger}$, Francesco Vitale ${ }^{1,2}$, \\ Claudio Pulvirenti ${ }^{5}$, Giovanni Rezza ${ }^{6}$, Fabrizio Vitale ${ }^{3}$, Giuseppa Purpari ${ }^{7}$, \\ Carmelo Massimo Maida ${ }^{1,2}$, Salvatore Zichichi ${ }^{5}$, Silvia Scibetta ${ }^{3}$, Walter Mazzucco ${ }^{1,2,8 *}$ \\ and Paola Stefanelli ${ }^{4}$
}

'Department of Health Promotion, Mother and Child Care, Internal Medicine and Medical Specialties "G. D'Alessandro", University of Palermo, Palermo, Italy, ${ }^{2}$ Regional Reference Laboratory of Western Sicily for the Emergency of COVID-19, Clinical Epidemiology Unit, University Hospital "Paolo Giaccone", Palermo, Italy, ${ }^{3}$ Molecular Biology Area, Zoo-prophilactic Experimental Institute of Sicily "A. Mirri", Palermo, Italy, ${ }^{4}$ Department of Infectious Diseases, National Health Institute, Rome, Italy, ${ }^{5}$ Uffici di sanità marittima, aerea e di frontiera (USMAF) - Servizi Assistenza Sanitaria Naviganti (SASN) Sicily, Ministry of Health, Directorate-General for Health Prevention, Rome, Italy, ${ }^{6}$ Directorate-General for Health Prevention, Ministry of Health, Rome, Italy, ${ }^{7}$ Virological Diagnostic Area, Zoo-prophilactic Experimental Institute of Sicily "A. Mirri", Palermo, Italy, ${ }^{8}$ Division of Biostatistics and Epidemiology, Cincinnati Children's Hospital Medical Centre, Cincinnati, OH, United States

Many African countries, representing the origin of the majority of refugees, asylum-seekers, and other migrants, toward regions bordering on the Mediterranean area, are experiencing sustained local transmission of severe acute respiratory syndrome coronavirus 2 (SARS-CoV-2). Sicily is one of the main entry gates of migrants crossing into Europe. We conducted a pilot study, based on the full-genome sequencing of SARS-CoV-2 strains isolated from migrants coming to Sicily by crossing the Mediterranean Sea, with the aim to investigate the viral genome polymorphism and to describe their genetic variations and the phylogenetic relationships. On June 21, a nongovernmental organization vessel rescued 210 migrants crossing the Mediterranean Sea from Libya to Sicily. Of them, 13.4\% tested positive for SARS-CoV-2. Eighteen whole genome sequences were obtained to explore viral genetic variability. All but one of the sequences clustered with other viral African strains within the lineage $A$, whereas only one intermixed among B.1 lineage genomes. Our findings documented that most of the investigated migrants acquired SARS-CoV-2 infection before landing in Sicily. However, SARS-CoV-2 transmission during travel or in overcrowded Libyan immigrant camps and/or illegal transport boats could not be ruled out. SARS-CoV-2 molecular surveillance on migrants arriving in Europe through the Sicilian gate may improve the knowledge of global SARS-CoV-2 transmission dynamic also in light of the emergence of new variants.

Keywords: SARS-CoV-2, molecular surveillance, migrant, asylum-seeker, Mediterranean Sea, NGS

\section{INTRODUCTION}

Although South Africa bears the greatest burden of disease in the continent with more than half of documented cases, other African countries, such as Nigeria, Ghana, Cameroon, Côte d'Ivoire, and Senegal, representing the origin of the majority of refugees, asylum-seekers, and other migrants toward regions bordering the Mediterranean 
area, are experiencing sustained local transmission of severe acute respiratory syndrome coronavirus 2 (SARS-CoV-2) $(1,2)$.

Sicily, being the closest territory to African borders by way of its smaller islands such as Lampedusa, is one of the first destinations of migrants crossing into Europe (3) (Supplementary Figure 1).

Between January 1, 2020 and October 21, 2020, 26,532 migrants/refugees landed in Sicily following the Libyan route by boat, either directly or after being rescued in the sea by Italian authorities or nongovernmental organizations (3), and were then hosted in dedicated reception camps or reconverted cruise ships.

Here, we report results from a pilot study based on the full-genome sequencing of SARS-CoV-2 strains isolated from migrants coming to Sicily by crossing the Mediterranean Sea in order to investigate the viral genome polymorphism, the genetic variations, and the phylogenetic relationships.

\section{METHOD}

\section{Study Population}

On June 21, a nongovernmental organization (NGO) rescue vessel saved 210 migrants near the Libyan border and arrived at the harbor of Porto Empedocle, in Southern Sicily. Of the 210 migrants, 68 (32.3\%) were children or adolescents. One of the migrants, presenting with fever and respiratory symptoms, was under treatment for $\mathrm{TB}$ and transferred to a hospital. A rhino-pharyngeal swab, collected at hospital admission, resulted positive for SARS-CoV-2 molecular testing. Quarantine measures were implemented, and after molecular screening, 28 (13 men, nine women, and six children) out of the remaining 209 migrants resulted positive (13.4\%). Of them, eight were from Cameroon, five from Guinea Conakry, three from Mali, two from Côte d'Ivoire, Sierra Leone, and Somalia, and 1 each from Nigeria, Togo, Senegal, Ghana, Liberia, and Bangladesh. The median age was 24 years. None of the migrants presented or developed signs or symptoms suggestive of COVID-19 during the follow-up.

\section{Ethical Approval}

This study was conducted with the approval of the ethics committee of Palermo University Hospital, Palermo, Italy (n. $7 / 2020$ released on $13 / 07 / 2020$ ), and it is in agreement with the Helsinki Declaration.

\section{SARS-CoV-2 Detection and Whole Genome Sequencing}

Total RNA was extracted by NucleoMag Virus (MachereyNagel, Germany) following the manufacturer's instructions and employing the KingFisher automatic nucleic acid extractor. SARS-CoV-2 specific targets, N1 and N2, were detected by real-time reverse transcriptase (RT)-PCR adopting primers and protocol published by the Centers for Disease Control and Prevention (CDC-006-00019, Revision: 02) (4). The probes were labeled with FAM-BHQ. PCR reactions were carried with the Brilliant III Ultra-Fast QRT-PCR Master Mix (Agilent, USA) using a QuantStudio 7 Pro Real-Time thermocycler (Thermo Fisher Scientific). Next generation sequencing (NGS) library was constructed by amplicon technique (5). Primers adopted for genome sequencing comprehended two pools, specially designed from Thermo Fisher Scientific, covering the entire genome of SARS-CoV-2. These primers are included in a package supplied by Illumina for AmpliSeq protocol (Document no. 1000000036408 v09) (5). The prepared library was purified and sequenced on MiSeq platform (Illumina). The fastq files were quality filtered and reads mapped with Bowtie2 software, against the reference genome from Wuhan (GenBank accession number NC_045512.2), to achieve the complete genome sequences. Clean genome data were visualized by IGV 2.8.0 software in order to investigate single nucleotide polymorphisms (SNPs) motives. The potentially resulting variable amino acids (AAs) in derived proteins compared to the Wuhan reference were investigated for the genomes retrieved in this study by visual inspection of the alignments.

\section{Phylogenetic Analysis}

To explore the lineages of viruses currently circulating in the populations in the study, a selection of 18 SARS-CoV2 genomes were obtained and analyzed, as first, through the "Pangolin COVID-19 Lineage Assigner" (6) in order to assign the lineages based on the methodology described in Rambaut (7). The assignment of the clade was also performed according to Nexstrain (8) classification. The genomes were analyzed in a phylogenetic context together with SARS-CoV-2 complete genomes from different countries, retrieved from GISAID (9) and GenBank (10), also including the above-mentioned reference sequence of the isolate Wuhan-Hu-1. Multiple nucleotide sequence alignment was performed using MAFFT v.7 (11) with the Galaxy platform $(12,13)$, and it was manually edited by Bioedit program (14).

The best fitting substitution model, together with the maximum likelihood (ML) phylogenetic tree, were obtained with Phyml v3.0 $(14,15)$. Support for the tree topology and clades was estimated with the Bayesian-like transformation of aLRT (aBayes) $(16,17)$. A maximum likelihood (ML) phylogenetic tree was also built with IQ-TREE software by using SH-a LRT and 1,000 number of replicates (18).

\section{RESULTS}

Overall, the RT-PCR assay showed SARS-CoV-2 targets with $\mathrm{Ct}$ values ranging from 16 to 36 . All samples were included in the next massive sequencing protocol, but suitable genome libraries were recovered from 18 samples with $\mathrm{Ct}$ value $<34$. Samples showed clean mapped reads with an average coverage of the genome (referred to NC_045512.2) ranging from 158 to about 1,000. Sequencing results and coverage did not appear particularly affected by any difference of the initial Ct values.

The lineage analysis showed that the majority of the sequences from migrants $(17 / 18,94.4 \%)$ belonged to lineage $A$, while only one sequence, named EPI_ISL_582768, belonged to lineage B.1. More in depth, the clade assignment revealed that the 17 genomes belonged to clade 19B and the remaining EPI_ISL_582768 viral strain belonged to $19 \mathrm{~A}$ clade.

The maximum likelihood phylogenetic tree is reported as a whole in Supplementary Figure 2. Figure 1 highlights selected 


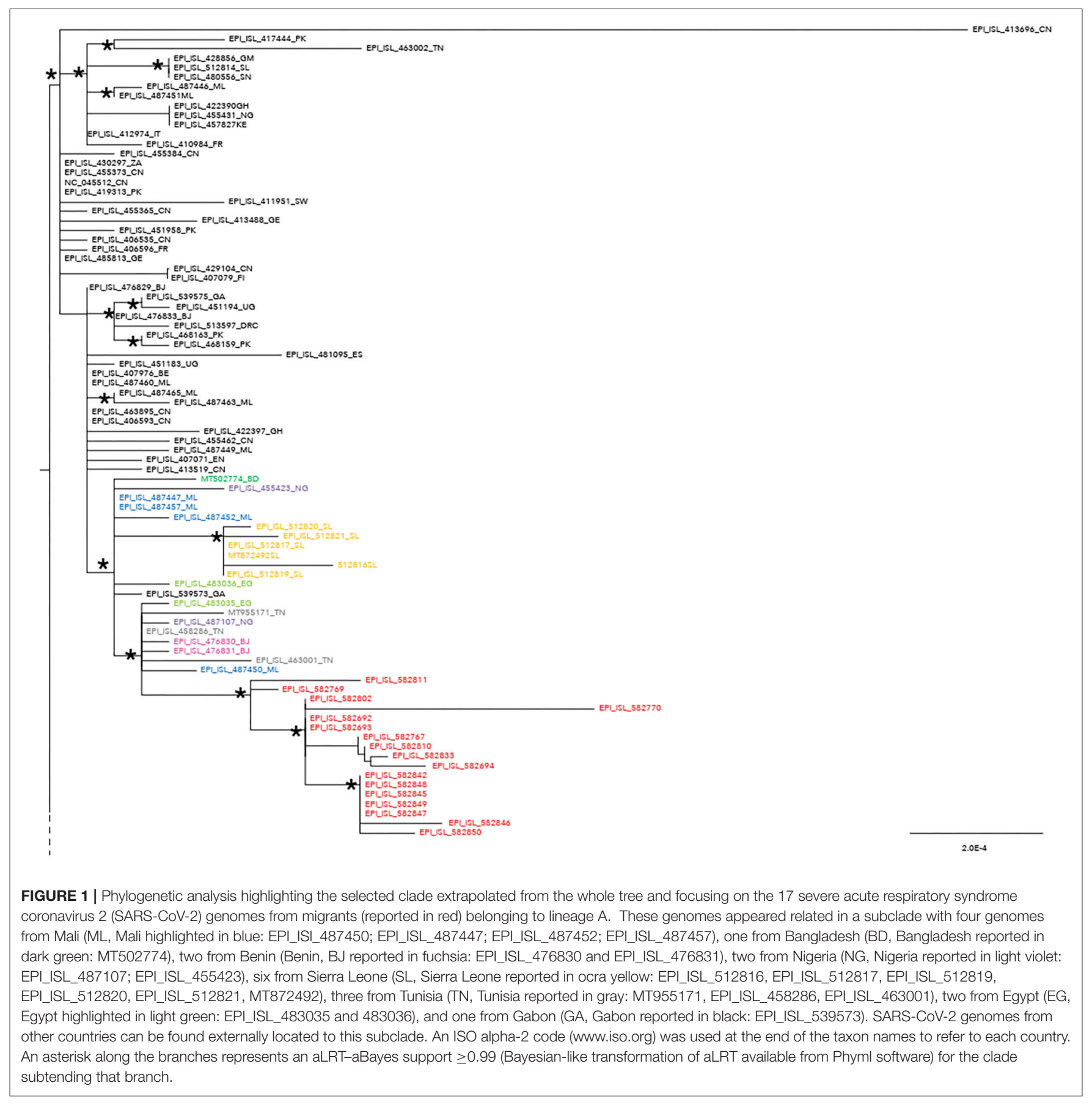

clades extrapolated from the whole tree including the genomes from migrants (reported in red) and belonging to lineage A (Figure 1) and lineage B.1 (Figure 2), respectively. The ML tree obtained with IQ-TREE confirmed the phylogenetic relationships above described (data not shown).

Fourteen SNPs were identified in $100 \%$ of the genomes, five of which did not involve an amino acid change and one felt into the $3^{\prime}$ untranslated region (UTR) region (Table 1). Amino acid mutations were located in $n s p 3$ (1062I, 1431V, 1612A, 1870F), in the RNA-dependent RNA polymerase (246I), ORF8 (84S), and nucleocapsid phosphoprotein (202N, 276K) (Table 1). Two SNPs were identified in $50 \%$ of the observed sequences, generating the mutations $313 \mathrm{~F}$ in $n s p 3$ and $45 \mathrm{~V}$ in $n s p 8$ (Table 1).

Seven SNPs were identified in only one genome $(7 \%, n=$ $1 / 14$ ), and four of these have not determined AA changes. Among those involving AA variations, we found the substitutions $49 \mathrm{H}$ and 208Y in nsp2 and 1009I in the spike (Table 1).

We also analyzed which of the SNPs identified in the genomes from migrants were also present in the genomes from the African countries highlighted by colors (Figure 1) and located in the 


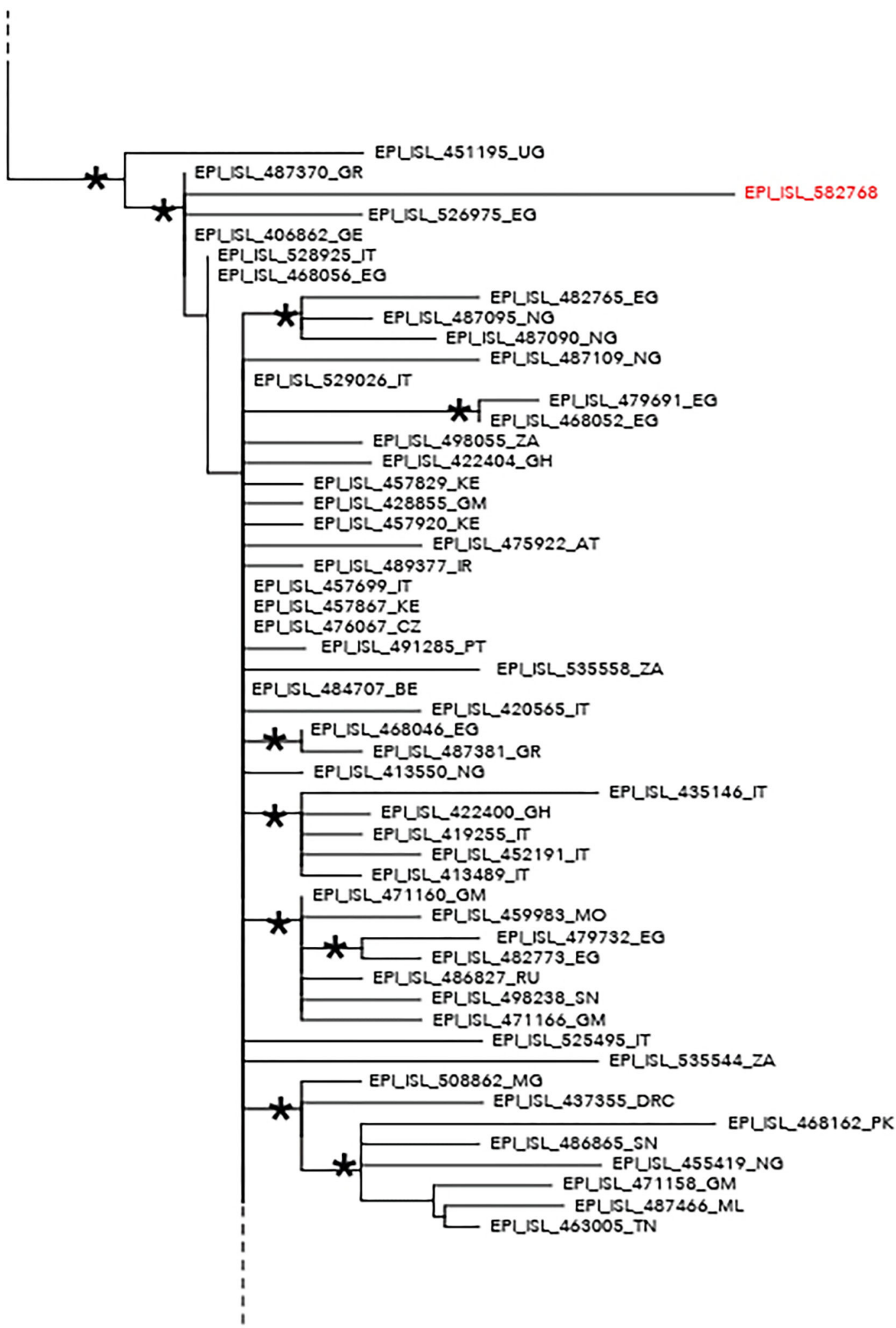

FIGURE 2 | Phylogenetic analysis highlighting the selected clade extrapolated from the whole tree and including the severe acute respiratory syndrome coronavirus 2 (SARS-CoV-2) strain EPI_ISL_582768 (highlighted in red) intermixed among other SARS-CoV-2 B-B.1 lineage genomes. This strain appeared intermixed among other genomes from different countries and more proximal to strains from Egypt (EPI_ISL_526975), Greece (EPI_ISL_487370), Germany (EPI_ISL_406862), and Uganda 
FIGURE 2 | (EPI_ISL_451195). The ISO alpha-2 codes (www.iso.org) were used at the end of the taxon names to refer to each country. An asterisk along the branches represents an aLRT-aBayes support $\geq 0.99$ (Bayesian-like transformation of aLRT available from Phyml software) for the clade subtending that branch.

TABLE 1 | The mutation points observed in 14 of the total 18 samples (partial genomes are not included in this detection).

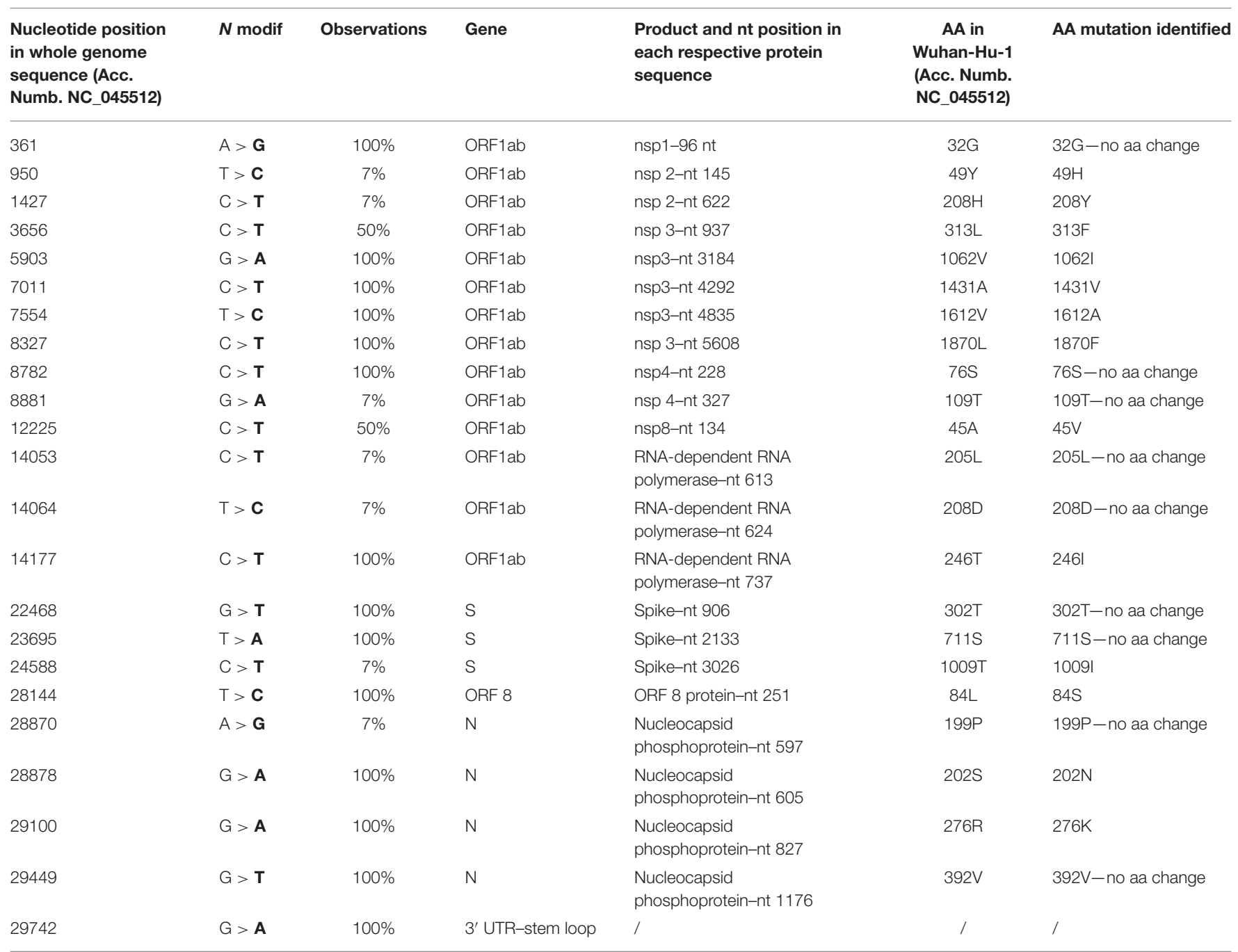

Bold values means modified nucleotides.

same supported subclade. We therefore found three specific SNPs confirmed also in the genomes from the African countries. In particular, SNPs at the nucleotide (nt) position $361(38 \%, n=$ $8 / 21), 8782(95 \%, n=20 / 21)$, and $22,468(95 \%, n=20 / 21)$.

The EPI_ISL_582768 revealed five SNPs that did not cause AA changes: one was located inside the $5^{\prime}$ UTR; meanwhile, four SNPs determined AA change (Table 2). Among those involving AA change, the first determined the mutation $21 \mathrm{M}$ in $n s p 3$, the second the mutation $216 \mathrm{~F}$ in $n s p 3$, the third the mutation $277 \mathrm{~S}$ in $n s p 6$, and the fourth the mutation 614G in the spike (Table 2).

\section{DISCUSSION}

The first case of COVID-19 was reported in the African continent on February 14, 2020 (19). Nevertheless, because of low-toabsent testing capacity and poor reporting systems, to date, limited information are available on the burden of COVID-19 and the genetic characteristics of SARS-CoV-2 viruses circulating in Africa (2, 20).

We investigated the viral genome polymorphism of SARSCoV-2 genomes isolated from a sample of migrants coming to Sicily by crossing the Mediterranean Sea, following the Libyan route, and hosted in dedicated reception centers (21). Our analysis identified some genomic lineages previously detected in different low-income countries. In particular, the majority of the genomes here investigated from migrants belonged to lineage A (only one sequence belonged to lineage B.1).

Despite the several limitations related to the convenient sample and to the lack of available genomes from each African country, phylogenetic relationships and SNPs analyses were carried out. 
TABLE 2 | The mutation points observed in the sample EPI_ISL_582768.

\begin{tabular}{|c|c|c|c|c|c|}
\hline $\begin{array}{l}\text { Nucleotide position in } \\
\text { whole genome sequence } \\
\text { (Acc. Numb. NC_045512) }\end{array}$ & $N$ modif & Gene & $\begin{array}{l}\text { Product and nt position } \\
\text { in each respective } \\
\text { protein sequence }\end{array}$ & $\begin{array}{l}\text { AA in Wuhan-Hu-1 } \\
\text { (Acc. Numb. } \\
\text { NC_045512) }\end{array}$ & AA mutation identified \\
\hline 241 & $\mathrm{C}>\mathbf{T}$ & 5' UTR & / & / & / \\
\hline 475 & $C>\mathbf{T}$ & ORF1ab & nsp1-nt 210 & $70 F$ & 70F-no aa change \\
\hline 2780 & $G>A$ & ORF1ab & nsp 3- nt 61 & $21 \mathrm{~V}$ & $21 \mathrm{M}$ \\
\hline 3037 & $C>\mathbf{T}$ & ORF1ab & nsp3-nt 318 & $106 \mathrm{~F}$ & 106F-no aa change \\
\hline 3365 & $C>\mathbf{T}$ & ORF1ab & nsp 3-nt 646 & $216 L$ & $216 F$ \\
\hline 7210 & $A>\mathbf{G}$ & ORF1ab & nsp3-nt 4491 & $1497 \mathrm{~K}$ & 1497K-no aa change \\
\hline 11801 & $G>A$ & ORF1ab & nsp6-nt 829 & $277 G$ & $277 S$ \\
\hline 22882 & $\mathrm{~T}>\mathbf{C}$ & $S$ & Spike-nt 1320 & $440 \mathrm{~N}$ & $440 \mathrm{~N}-$ no aa change \\
\hline 23403 & $A>\mathbf{G}$ & S & Spike-nt 1841 & $614 \mathrm{D}$ & $614 G$ \\
\hline 26936 & $\mathrm{C}>\mathbf{T}$ & $\mathrm{M}$ & $\begin{array}{l}\text { Membrane glycoprotein-nt } \\
414\end{array}$ & $138 \mathrm{~L}$ & 138L-no aa change \\
\hline
\end{tabular}

Bold values means modified nucleotides.

Phylogenetic analysis consistently placed the migrant genomes, except for one, in a supported subclade grouping with viral African genomes (lineage A) identified in Mali, Bangladesh, Benin, Nigeria, Sierra Leone, Tunisia, Egypt, and Gabon. The EPI_ISL_582768 clustered in a different clade, intermixed among B-B.1 lineage genomes from various countries, and more proximal to strains from Egypt, Greece, Uganda, and Germany.

The unique sample clustering among B-B.1 lineage genomes exhibited a signature mutation profile near to ST4 [previously described in Yang et al. (22)] that includes three SNPs: C241T, C3037T, and A23403G. In Africa, ST4 has been reported for cases reporting travel history to Europe (23). Moreover, lineage B.1 was described in some African countries, due to returning travelers (20). As reported, several hypotheses could be in support to the origin of the infection of the only one genome belonging to the B.1 lineage.

In agreement with previous data $(8,9,24,25)$, we highlighted in the lineage A isolates from migrants the two very stable SNPs, i.e., C8782T and T28144C, previously reported to be marker variant and specific of clade S-lineage A (7). This finding is consistent with the highest frequencies of lineage A previously reported in Africa (93\%) (25).

The genetic variability due to the presence of SNPs associated with the different important encoding proteins, have been, at least in part, previously reported $(8,9,26-28)$. Most of them have to be carefully monitored as a crucial role in the evolution of SARS-CoV-2. Specifically, mutations in the spike gene and in the RNA-dependent RNA polymerase may have a role as target for vaccine design and antiviral treatment.

Overall, we hypothesize that migrants have acquired SARSCoV-2 infection before landing in Sicily. However, SARS-CoV-2 transmission during travel or in overcrowded Libyan immigrant camps and/or illegal transport boats could not be ruled out (29).

These findings support the use of extensive genomic surveillance of SARS-CoV-2 among asylum-seekers arriving in Italy through the Sicilian gate also in light of the emergence of new variants (30). Migrant reception camps may provide an opportunity to improve knowledge on SARS-CoV-2 dynamic in "neglected" geographical areas and on genetic diversity and phylogenetic relationships in order to improve prevention and control programs for vulnerable populations $(31,32)$.

Lastly, the study of virus genetic variations in poorly resourced countries and their evolutionary trajectories may be useful for global SARS-CoV-2 transmission dynamics (20).

\section{DATA AVAILABILITY STATEMENT}

The original contributions presented in the study are publicly available. This data can be found here: The genome sequences were deposited into GenBank database with accession numbers from MW340787 to MW340802.

\section{ETHICS STATEMENT}

The studies involving human participants were reviewed and approved by Ethics committee of Palermo University Hospital, Palermo, Italy. Written informed consent from the participants' legal guardian/next of kin was not required to participate in this study in accordance with the national legislation and the institutional requirements.

\section{AUTHOR CONTRIBUTIONS}

FT, FrV, FaV, WM, and PS: methodology. SR, SS, and AL: formal analysis. All authors: investigation and writing, review, and editing.

\section{ACKNOWLEDGMENTS}

We gratefully acknowledge the authors and the originating and submitting laboratories for their sequence and metadata shared through GISAID and NCBI, on which this research is based. All submitters of data may be contacted directly via www.gisaid.org. The Acknowledgments Table for GISAID and NCBI is reported as Supplementary Table 1. The authors thank Dr. Angela Di Martino, Dept. Infectious Diseases, Istituto Superiore di Superiore for the helpful assistance. 


\section{SUPPLEMENTARY MATERIAL}

The Supplementary Material for this article can be found online at: https://www.frontiersin.org/articles/10.3389/fpubh. 2021.632645/full\#supplementary-material

Supplementary Figure 1 | Main route followed by migrants to Europe through the Sicilian gate.

Supplementary Figure 2 | Maximum likelihood phylogenetic analysis of 18 SARS-CoV-2 genomes from migrants (reported in red) and 262 SARS-CoV-2 complete genomes from different countries, retrieved from GISAID and GenBank

\section{REFERENCES}

1. COVID-19 Dashboard by the Center for Systems Science and Engineering (CSSE) at Johns Hopkins University (JHU). Available online at: https:// coronavirus.jhu.edu/map.html (accessed October 26, 2020).

2. WHO African Region. Available online at: https://apps.who.int/iris/bitstream/ handle/10665/335766/SITREP_COVID-19_WHOAFRO_20200930-eng.pdf (accessed October 26, 2020).

3. Department of Public Security of the Ministry of Internal Affairs of the Italian Republic. Available online at: https:/www.interno.gov.it/sites/default/files/ 2020-10/cruscotto_statistico_giornaliero_01-10-2020.pdf (accessed October 26, 2020).

4. Centers for Disease Control and Prevention - Division of Viral Disease. Available online at: https://www.fda.gov/media/134922/download (accessed October 26, 2020).

5. AmpliSeq for Illumina On-Demand, Custom, and Community Panels. Reference Guide (Document \# 1000000036408 v09). Illumina Proprietary (2020). Available online at: https://support.illumina.com/content/dam/ illumina-support/documents/documentation/chemistry_documentation/ ampliseq-for-illumina/ampliseq-for-illumina-custom-and-communitypanels-reference-guide-1000000036408-09.pdf (accessed January 30, 2021).

6. Pangolin COVID-19 Lineage Assigner. Available online at: https://pangolin. cog-uk.io (accessed October 26, 2020).

7. Rambaut A, Holmes E, Hill V, O’Toole A, McCrone JT, Ruis C, et al. A dynamic nomenclature proposal for SARSCoV-2 to assist genomic epidemiology. Nat Microbiol. (2020) 5:1403-7. doi: 10.1101/2020.04.17.046086

8. Nexstrain. Available online at: https://clades.nextstrain.org (accessed October 26, 2020).

9. GISAID. Available online at https://www.gisaid.org (accessed October 26, 2020).

10. GenBank - NCBI. Available online at: https://www.ncbi.nlm.nih.gov/pubmed (accessed October 26, 2020).

11. Katoh K, Standley DM. MAFFT multiple sequence alignment software version 7: improvements in performance and usability. Mol Biol Evol. (2013) 30:77280. doi: $10.1093 / \mathrm{molbev} / \mathrm{mst} 010$

12. Galaxy Platform. Available online at: https://usegalaxy.org (accessed October 26, 2020).

13. Afgan E, Baker D, Batut B, Van Den Beek M, Bouvier D, Cech M, et al. The galaxy platform for accessible, reproducible and collaborative biomedical analyses: 2018 update. Nucleic Acids Res. (2018) 46:W53744. doi: 10.1093/nar/gky379

14. Hall T. BioEdit: a user-friendly biological sequence alignment editor and analysis program for windows 95/98/NT. Nucleic Acids Symp Ser. (1999) 41:5-8.

15. Lefort V, Longueville J-E, Gascuel O. SMS: smart model selection in PhyML. Mol Biol Evol. (2017) 34:2422-4. doi: 10.1093/molbev/msx149

16. Guindon S, Dufayard JF, Lefort V, Anisimova M, Hordijk W, Gascuel O. New algorithms and methods to estimate maximum-likelihood phylogenies: assessing the performance of PhyML 3.0. Syst Biol. (2010) 59:30721. doi: $10.1093 /$ sysbio/syq010

17. Anisimova M, Gil M, Dufayard JF, Dessimoz C, Gascuel O. Survey of branch support methods demonstrates accuracy, power, and robustness of fast likelihood-based approximation schemes. Syst Biol. (2011) 60:68599. doi: 10.1093/sysbio/syr041

18. Trifinopoulos J, Nguyen LT, von Haeseler A, Minh BQ. W-IQ-TREE: a fast online phylogenetic tool for maximum likelihood analysis. Nucl Acids Res. (2016) 44:W232-5. doi: 10.1093/nar/gkw256 obtained with the best fitting substitution model with Phyml v3.0. The tree was midpoint rooted. The scale bar at the bottom of the tree represents 0.0002 nucleotide substitution per site. The ISO alpha-2 codes (www.iso.org) were used at the end of the taxon names to refer to each country. An asterisk along the branches represents an aLRT - aBayes support $\geq 0.99$ (Bayesian-like transformation of aLRT available from Phyml software) for the clade subtending that branch. The African genomes located in the sub-clade in the upper part of the tree were highlighted in different colors (as described

in Figure 1)

Supplementary Table 1 | Sequences used in this study and acknowledgment table.

19. Umviligihozo G, Mupfumi L, Sonela N, Naicker D, Obuku EA, Koofhethile C, et al. Sub-Saharan Africa preparedness and response to the COVID-19 pandemic: a perspective of early career African scientists. Wellcome Open Res. (2020) 5:163. doi: 10.12688/wellcomeopenres.16070.2

20. Costantino C, Cannizzaro E, Alba D, Conforto A, Cimino L, Mazzucco W. Sars-Cov-2 pandemic in the mediterranean area: epidemiology and perspectives. EuroMediterranean Biomed J. (2020) 15:102-6. doi: 10.3269/1970-5492.2020.15.25

21. European Parliament. Available online at: https://www.europarl.europa.eu/ factsheets/it/sheet/152/politica-d-immigrazione (accessed October 26, 2020).

22. Yang X, Dong N, Chan EW-C, Chen S. Identification of super-transmitters of SARS-CoV-2. Available online at: https://www.medrxiv.org/content/10. $1101 / 2020.04 .19 .20071399 v 1$

23. Bugembe DL, Kayiwa J, Phan MV, Tushabe P, Balinandi S, Dhaala B, et al. Main routes of entry and genomic diversity of SARS-CoV-2, Uganda. Emerg Infect Dis. (2020) 26:2411-5. doi: 10.3201/eid2610.202575

24. Tang $\mathrm{X}, \mathrm{Wu} \mathrm{C}$, Li $\mathrm{X}$, Song $\mathrm{Y}$, Yao $\mathrm{X}, \mathrm{Wu} \mathrm{X}$, et al. On the origin and continuing evolution of SARS-CoV-2. Natl Sci Rev. (2020) 7:101223. doi: $10.1093 / \mathrm{nsr} /$ nwaa036

25. Gómez-Carballa A, Bello X, Pardo-Seco J, Martinón-Torres F, Salas A. Mapping genome variation of SARS-CoV-2 worldwide highlights the impact of COVID-19 super-spreaders. Genome Res. (2020) 30:143448. doi: $10.1101 /$ gr.266221.120

26. Pachetti M, Marini B, Benedetti F, Giudici F, Mauro E, Storici P, et al. Emerging SARS-CoV-2 mutation hot spots include a novel RNA-dependent-RNA polymerase variant. J Transl Med. (2020) 18:179. doi: 10.1186/s12967-020-02344-6

27. Lo Presti A, Rezza G, Stefanelli P. Selective pressure on SARS-CoV-2 protein coding genes and glycosylation site prediction. Heliyon. (2020) 6:e05001. doi: 10.1016/j.heliyon.2020.e05001

28. Mercatelli D, Giorgi FM. Geographic and genomic distribution of SARS-CoV2 mutations. Front Microbiol. (2020) 11:1800. doi: 10.3389/fmicb.2020.01800

29. Daw M, El-Bouzedi A, Ahmed M. COVID-19 and African immigrants in North Africa: a hidden pandemic in a vulnerable setting. Disast Med Public Health Prepared. (2020) 19:1-2. doi: 10.1017/dmp.2020.387

30. Makoni M. South Africa responds to new SARS-CoV-2 variant. Lancet. (2021) 397:267. doi: 10.1016/S0140-6736(21)00144-6

31. Tramuto F, Mazzucco W, Maida CM, Affronti A, Affronti M, Montalto G, et al. Serological pattern of Hepatitis B, C, and HIV infections among immigrants in Sicily: epidemiological aspects and implication on public health. J Community Health. (2012) 37:547-53. doi: 10.1007/s10900-011-9477-0

32. Ceccarelli G, Lopalco M, d'Ettorre G, d'Ettorre G, Ciccozzi M. Surveillance of COVID-19 in migrant reception centers: a call for action. J Travel Med. (2020) 18:taaa171. doi: 10.1093/jtm/taaa171

Conflict of Interest: The authors declare that the research was conducted in the absence of any commercial or financial relationships that could be construed as a potential conflict of interest.

Copyright (C) 2021 Tramuto, Reale, Lo Presti, Vitale, Pulvirenti, Rezza, Vitale, Purpari, Maida, Zichichi, Scibetta, Mazzucco and Stefanelli. This is an open-access article distributed under the terms of the Creative Commons Attribution License (CC $B Y)$. The use, distribution or reproduction in other forums is permitted, provided the original author(s) and the copyright owner(s) are credited and that the original publication in this journal is cited, in accordance with accepted academic practice. No use, distribution or reproduction is permitted which does not comply with these terms. 